\title{
QUALITY COMPARISON AND ACCELERATION FOR DIGITAL HOLOGRAM GENERATION METHOD BASED ON SEGMENTATION
}

\author{
Hoonjong Kang, Fahri Yaraş, and Levent Onural \\ Dept. of Electrical and Electronics Engineering, Bilkent University, \\ TR-06800 Bilkent, Ankara, Turkey \\ hjkang@ee.bilkent.edu.tr, fahri@ee.bilkent.edu.tr, onural@ee.bilkent.edu.tr
}

\begin{abstract}
A holographic fringe pattern generation methods is based on Fraunhofer diffraction and subsequent segmentation and approximation of the fringe pattern. Several modifications of the original algorithm are already proposed to improve the quality of reconstructions. We compare the quality of to the reconstructed images from different versions of this algorithm by taking the reconstructions from the Fresnel hologram as a reference. Since, there is not any generally accepted objective quality assessment method for such reconstructions, we used some experimental methods such as intensity spread over the reconstructed images, total noise power, and peak-signal-tonoise for comparison. Then we chose the best performing algorithm in terms of ireconstruction quality, and developed a GPU-based implementation to accelerate the computation speed. The quality of the resultant reconstructions is comparable to reconstructions from Fresnel holograms; much higher speed is achieved due to multi-GPU implemetation.
\end{abstract}

Index Terms - Digital holography, real-time holography, Fresnel hologram, graphic processing unit, objective quality assessment, digital hologram reconstruction

\section{INTRODUCTION}

Electro-holography often requires tens of seconds of computation time to generate the holographic fringe pattern. There are several ways to solve this computational problem such as using hardware instead of software [1, 2, 3], using better algorithms or finding different physical models [4]. The coherent holographic stereogram (CS) [5] is a fast method which significantly reduces the computational complexity. The main algorithm in this approach uses discrete spatial frequencies and the associated phases. Modified and improved versions of the basic stereogram are reported in the literature $[6,7$, $8,9,10,11,12]$. In this paper, quality improvements due to the phase-added stereogram (PAS), which is the basic version of the CS, are described. The quality of reconstructions

This work is supported by EC within FP7 under Grant 216105 with the acronym Real 3D. is compared to the quality of reconstruction from the Fresnel hologram. Furthermore, the accurate phase-added stereogram (ACPAS), latest version, is implemented on a multi GPU environment, and thus, a higher speed is achieved.

\section{QUALITY IMPROVEMENTS FOR COHERENT STEREOGRAM}

Computational speed of the CS is much faster than that of the Fresnel hologram due to the segmentation of the output fringe pattern and inverse fast Fourier transform (IFFT). The calculation of the CS comprises two steps. In the first step the output fringe pattern is divided into suitable square size segments.Each segment is composed of superposition of weighted 2D complex sinusoids which are the contributions of object points to the hologram pattern over that segment. Complex amplitudes of the sinusoids depend on the position and amplitude of 3D object points. In a second step each such superposed pattern is transformed by IFFT. Repeating this procedure for each segment completes the computation. In a second step, the frequency plane is discretized, and the original frequency components are modified by changing their frequencies to the value of the nearest allowed discrete frequency; the complex amplitudes of the original frequency components are kept unaltered. This modification is a source of error (noise) on the reconstruction.

The compensated phase-added stereogram (CPAS), accurate phase-added stereogram (APAS), and ACPAS are improved versions of the PAS. The CPAS is to compensate the error caused by the mapping to discrete value by means of adding the phase compensation, which is computed from the differences between the spatial frequency values in the continuous and the discrete domains. The reconstructed image of the CPAS is better than that of the PAS. The calculation time is almost the same as the PAS. The calculation of the CPAS is described below. The fundamental spatial frequency component of a fringe pattern in a segment, in cycles per unit length, is,

$$
f_{F S F}=\frac{1}{T_{F P}}=\frac{1}{\Delta S}=\frac{1}{\Delta P N_{s}},
$$

where $T_{F P}$ is the fundamental period, $\Delta S$ is the segmenta- 
tion size, $\Delta P$ is the pixel size, and $N_{s}$ is the number of pixels along one direction of a segment. We take the number of pixels in a segment as $N_{s} \times N_{s}$. Accordingly, the coordinates of the spatial frequencies are $\xi_{\text {int }}=\operatorname{integer}\left(f_{p \xi c} / f_{F S F \xi}\right)$, and $\eta_{\text {int }}=\operatorname{integer}\left(f_{p \eta c} / f_{F S F \eta}\right)$, where $f_{p \xi c}$ and $f_{p \eta c}$ are the continuous spatial frequencies on $\xi$ and $\eta$ axes, and $\xi_{\text {int }}$ and $\eta_{\text {int }}$ are the corresponding horizontal and vertical discrete coordinates. Here the integer $(\cdot)$ refers to the "nearest integer" operator. The spatial frequencies $f_{p \xi c}$ and $f_{p \eta c}$ are determined as $\left(\sin \theta_{p \xi c}-\sin \theta_{\xi \mathrm{ref}}\right) / \lambda$ and $\left(\sin \theta_{p \eta c}-\sin \theta_{\eta \mathrm{ref}}\right) / \lambda$, where $\theta_{p \xi c}$ and $\theta_{\xi \text { ref }}$ are the incidence angles of the object and reference beams on $\xi$ axis, and $\theta_{p \eta c}$ and $\theta_{\eta \mathrm{ref}}$ are the incidence angles on $\eta$ axis. The related frequency error compensations are $C_{\xi}=j 2 \pi\left(f_{p \xi c}-f_{p \xi c i n t}\right)$ and $C_{\eta}=j 2 \pi\left(f_{p \eta c}-f_{p \eta c i n t}\right)$

on the $\xi$ and $\eta$ axes, respectively. The spatial frequency and the phase at the center of a segment are used to calculate the PAS of the segment. Then the error due to discretization of the spatial frequencies is reduced by adding the error compensations to the phases. Consequently, the cosine function form of the compensated PAS for only real-term is computed as [9],

$$
\begin{aligned}
\mathbf{I}_{C P A S}(\xi, \eta) & =\sum_{p=1}^{N} \frac{a_{p}}{r_{p}} \cos \left\{j 2 \pi \left[\left(\xi-\xi_{c}\right) f_{p \xi \operatorname{cint}}\right.\right. \\
& \left.\left.+\left(\eta-\eta_{c}\right) f_{p \eta \operatorname{cint}}\right]+k r_{p}+C_{\xi}+C_{\eta}\right\},
\end{aligned}
$$

where $N$ is the number of object points. The wave number $k$ is $2 \pi / \lambda$, where $\lambda$ is the free-space wavelength of the coherent light. The distance $r_{p}$ between the $p$-th object point and the point $(\xi, \eta)$ on the hologram is $\left[\left(\xi-x_{p}\right)^{2}+(\eta-\right.$ $\left.\left.y_{p}\right)^{2}+z_{p}^{2}\right]^{1 / 2}$. Spatial frequencies $f_{p \xi \text { cint }}$ and $f_{p \eta c i n t}$ are determined as $f_{p \xi c i n t}=\xi_{\text {int }} f_{F S F \xi}$, and $f_{p \eta \text { int }}=\eta_{\text {int }} f_{F S F \eta}$, respectively.

In the computational procedures of the CS, if the segment size gets smaller, and if the IFFT size is taken to be equal to the segment size as in PAS, the discretization of the frequencies gets coarser. On the other hand, if the segment size gets larger, the approximation error which assumes a single frequency contribution from each object point also gets larger. The noise over the reconstructed image increases in both cases. There is an optimal choice of the segment size to minimize the overall error in the reconstructed image. The optimal segment size is determined as $\Delta S \leq(\Delta f)^{-1}$, where $\Delta f$ is the difference of spatial frequency between neighboring segments $[10,11,12]$. However, even if the segmentation size is determined by the above method, the quality is not increased since there is a trade-off as mentioned above. Nevertheless, this problem can be overcome by using a larger IFFT size per each segment.

The APAS uses a larger IFFT size without any phase compensation as the CPAS, and thus reduces the error due to discretized spatial frequency domain. In this case, the hologram segment is obtained by truncating the large-size IFFT output properly. As a consequence of the larger IFFT size, the quality of the reconstructed image using the APAS is close to the continuous-frequency PAS. However, the computing time of the APAS is longer than the discrete-frequency PAS due to larger size IFFT computations. Therefore, the IFFT size should be decided according to application. The ACPAS, latest version of the CS, is generated by using both fringe generation method of the CPAS and the APAS. Therefore, the ACPAS has both advantages: phase compensation and reduction of the error due to finer discretization at the spatial frequency domain. Consequently the ACPAS gets a higher quality reconstructed image than the other previous versions. In addition, the reconstruction from the ACPAS closely resemble the reconstruction from the Fresnel hologram.

\section{QUALITY COMPARISON}

A generated fringe pattern by the Fresnel model has a continues spatial frequency distribution. Therefore, the diffracted light by the fringe is steered to a designed direction. However, the generated fringe pattern by the CS has a discrete spatial frequency distribution. This causes noise on the reconstructed image because the diffracted light by the each segment on the fringe pattern may not be steered to the exact designed direction. In order to understand the effect of noise, the reconstructed images are compared to the reconstructed image from the Fresnel hologram. In this experiment, a single point located at the center position of a hologram display area is used as the input. Various methods are utilized such as Fresnel hologram, PAS without FFT, PAS with FFT, CPAS, APAS and ACPAS as the fringe generation algorithm $[6,9,11,12]$. The measured intensity distributions of the reconstructed images for each case are shown in Fig. 1. In the parenthesis of APAS and ACPAS notation, first and second terms mean IFFT size and segment size, respectively. As seen in Fig. 1, the Fresnel hologram and the PAS without FFT give a single peak without any noise. Here, the highest peak means the reconstructed object, and the other components are the noise which is caused by inaccurate superposition of the diffracted light. The other methods have a single high peak with some noise around it. The total noise levels of each reconstructed image are shown in Fig. 2. Although the reconstructed images of the improvements of the CS have some noise, these methods are acceptable fringe generation algorithms because they are much faster than that of the Fresnel hologram, and the noise may not be noticeable by the observer. These improvements, particularly the ACPAS and the CPAS, can be utilized for real-time holographic display systems, and can be selected according to the computational performance of a used system. The peak-signal-to-noise ratio (PSNR) is used as the objective quality assessment method. The mean squared error (MSE) which for two $m \times n$ reconstructed images $I_{r F H}$ and $I_{r C S}$ from the Fresnel hologram and the improvements, 


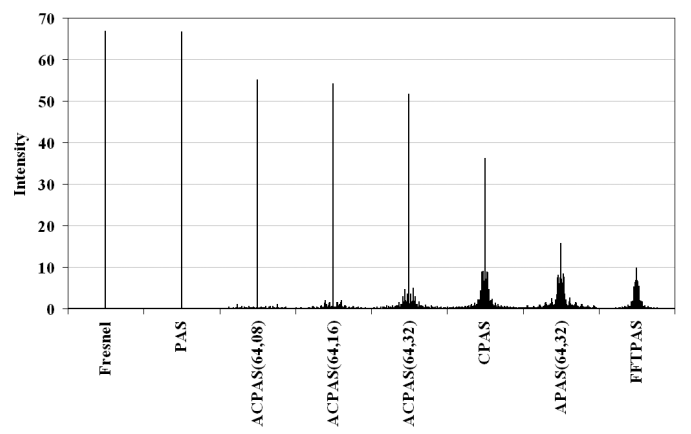

Fig. 1. Intensity distributions of reconstructed images.

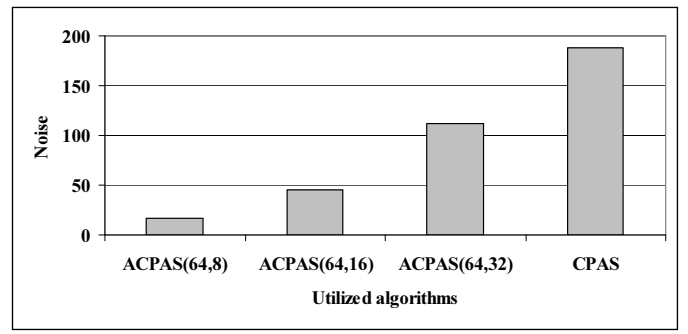

Fig. 2. Total power except the single peak.

respectively, is determined as,

$$
M S E=\frac{1}{m n} \sum_{i=0}^{m-1} \sum_{j=0}^{n-1}\left(I_{r F H}(i, j)-I_{r C S}(i, j)\right)^{2}
$$

and the PSNR is computed as,

$$
P S N R=10 \cdot \log _{10}\left(\frac{255^{2}}{M S E}\right) .
$$

The measured results are shown in Fig. 3. The ACPAS $(64,08)$ yields highest noise power among other tested algorithms. One interesting result is that even the reconstruction of the PAS with FFT has a low spread of peaks as shown in Fig. 1, it yields a higher noise level because not only peaks at center area but also whole reconstruction area, which is zero, also contributes to the PSNR. Therefore, a suitable objective quality assessment method for holography is superior and desirable.

\section{ACPAS ACCELERATION ON GPU}

A graphics processing unit (GPU) is a processor attached to a graphics card dedicated to calculating floating point operations. Modern GPUs are very efficient to use for scientific computing, because their highly parallel structure makes them more effective than general-purpose CPUs for a range of complex algorithms. The CS computes each segment separately, and all segments share the source input object points for this

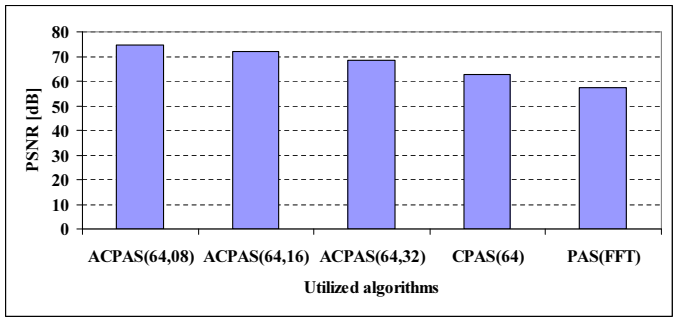

Fig. 3. Measured PSNR results corresponding to the reconstructed images of various fringes.

computation. Therefore, this show that the CS algorithm can be easily mapped to a single instruction multiple data architecture, and thus the ACPAS computing can significantly benefit from GPU based implementations.

In this work, the latest version ACPAS has been implemented based on multi-GPU using Compute Unified Device Architecture (CUDA) as a programming environment. The computation of the ACPAS is performed in three steps: calculation of spatial frequencies and phases in each segment, IFFT for each segment, and normalization of the complex fringe pattern. In order to accelerate the ACPAS computation, the three steps are implemented not on CPU but on GPU. The CPU just controls the GPU computing flow and data flow between the computer main memory and GPU memory. Computation times corresponding to the $\mathrm{CGH}$ generation algorithms for different number of object points are shown in Fig. 4. By the multi GPU computing environment, we could achieve up to 300 times faster computation compared to a typical CPU-based implementation.

One mega-pixel frame size full color holograms can be generated at a rate of 30 frames per second for objects with more than 10,000 points. Comparative results for the ACPAS on a CPU and on three GPUs are shown in Fig. 4. The used parameters in this experiment are as follow: the hologram size is $1 \mathrm{~K} \times 1 \mathrm{~K}$ pixels; the IFFT size is $64 \times 64$ pixels and the corresponding segment size is $32 \times 32$ pixels. The computing system has two Intel(R) Xeon(R) CPU 2GHz, 8-GByte memory and three NVIDIA GeForce GTX 280 video cards.

A 3D model, Rose, is used in this experiment as the input model, and this model is shown in Fig. 5(a). The used dense 3D object points are extracted from the 3D model, and are used to generate hologram patterns using the Fresnel hologram, the CPAS, and the ACPAS algorithms, for comparison. The reconstructed images are shown in Fig. 5(b) - 5(d). The used 3D object consisted of about 9,000 vertices, and therefore, the rendered images are photorealistic. The reconstructed image using the CPAS is not clear, and seems to have some noise. On the other hand, the quality of the reconstructed images using the ACPAS and the unapproximated Fresnel holograms are similar. 


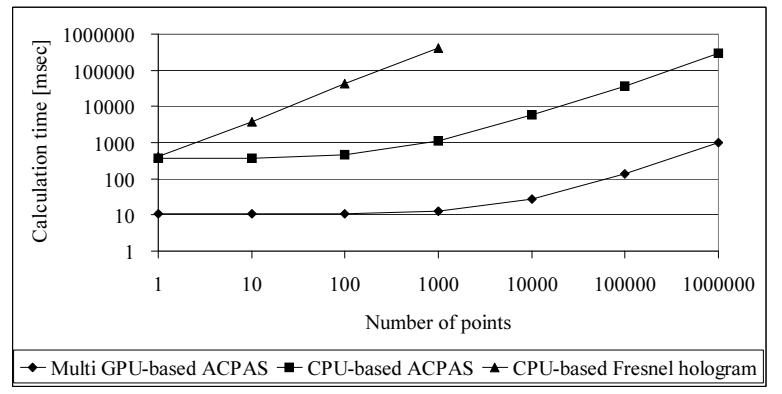

Fig. 4. Calculation-time comparison between CPU-based and GPU-based implementations.

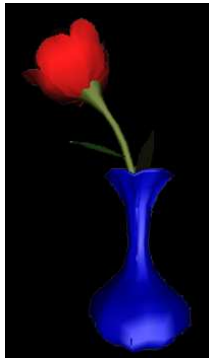

(a) 3D model

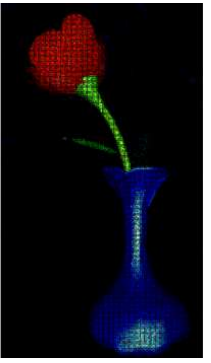

(b) Fresnel

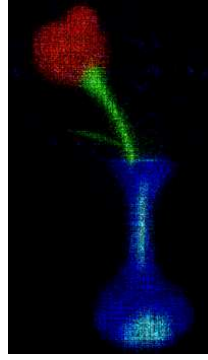

(c) CPAS

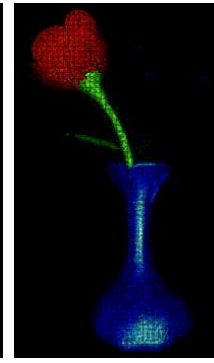

(d) ACPAS
Fig. 5. A perspective image of the used 3D model, Rose, and reconstructed images.

\section{CONCLUSION}

Various quality improvements for the CS are described. These improvements are due to phase compensation and reduction of frequency discretization step-size. The reconstructed images using these methods are compared to the reconstructed images from the Fresnel holograms, where a single point object is used as the input. The reconstructed images due to the improvements have a single high peak with lower unwanted components (noise). The latest version, ACPAS, has the highest peak with lowest noise. The reconstruction using the ACPAS is quite similar to the reconstruction from the Fresnel hologram. And even if it has some noise, this method is acceptable because it is much faster than that the Fresnel hologram case, and the noise can be neglected, since it is not noticeable.

Furthermore, the speed up due to multi-GPU implemented ACPAS is discussed. We can generate high quality hologram patterns in real-time as a consequence of a speed up factor of about 300 compared to CPU-based implementations. The digital holograms generated by the ACPAS can be computed and displayed at a rate of 30 fps for photorealistic scenes represented by ten thousands of object points.

\section{REFERENCES}

[1] T. Ito, N. Masuda, K. Yoshimura, A. Shiraki, T. Shimobaba, and T. Sugie, "Special-purpose computer horn-5 for a real-time electroholography," Opt. Express, vol. 13, pp. 1923-1932, March 2005.

[2] J. A. Watlington, M. Lucente, C. J. Sparrell, V. M. Bove, and I. Tamitani, "A hardware architecture for rapid generation of electro-holographic fringe patterns," in Practical Holography IX. SPIE, 1995, vol. 2406, pp. 172183.

[3] T. Okada, S. Iwata, O. Nishikawa, K. Matsumoto, H. Yoshikawa, K. Sato, and T. Honda, "The fast computation of holograms for the interactive holographic $3 \mathrm{~d}$ display system," in International Conference on Applications of Optical Holography. SPIE, 1995, vol. 2577, pp. 33-40.

[4] M. Lucente, "Interactive computation of holograms using a look-up table," Journal of Electronic Imaging, vol. 2, pp. 28-34, 1993.

[5] T. Yatagai, "Stereoscopic approach to 3-d display using computer-generated holograms," Appl. Opt., vol. 15, pp. 2722-2729, August 1976.

[6] M. Yamaguchi, H. Hoshino, T. Honda, and N. Ohyama, "phase-added stereogram: calculation of hologram using computer graphic technique," in Practical holographic VII. SPIE, 1993, vol. 1914, pp. 25-33.

[7] H. Yoshikawa and H. Kameyama, "Integral holography," in Practical Holograhy IX. SPIE, 1995, vol. 2406, pp. 226-234.

[8] J. Tamai and H. Yoshikawa, "Faster computation of subsampled coherent stereogram (in japanese)," Journal of ITEJ, vol. 50, pp. 1612-1615, 1996.

[9] H. Kang, T. Fujii, T. Yamaguchi, and H. Yoshikawa, "The compensated phase-added stereogram for realtime holographic display," Optical Engineering, vol. 46, pp. 095802, September 2007.

[10] H. Kang, T. Yamaguchi, and H. Yoshikawa, "Accurate phase-added stereogram to improve the coherent stereogram," Appl. Opt., vol. 47, pp. D44-D54, July 2008.

[11] H. Kang, Quality Improvements of the Coherent Holographic Stereogram for Natural 3D Display and Its Applications, Ph. D. Thesis, Nihon University, Japan, 2008.

[12] H. Kang, F. Yaraş, L. Onural, and H. Yoshikawa, "Realtime fringe pattern generation with high quality," in Digital Holography and Three-Dimensional Imaging. OSA, 2009, p. DTuB7. 